Journal Club

Editor's Note: These short, critical reviews of recent papers in the Journal, written exclusively by graduate students or postdoctoral fellows, are intended to summarize the important findings of the paper and provide additional insight and commentary. For more information on the format and purpose of the Journal Club, please see http://www.jneurosci.org/misc/ifa_features.shtml.

\title{
Jnk1 Activity is Indispensable for Appropriate Cortical Interneuron Migration in the Developing Cerebral Cortex
}

\author{
J. Jacob Riches and Kathryn Reynolds \\ Graduate Department of Molecular and Cellular Biology, College of Biological Science, University of Guelph, Guelph, Ontario, Canada, N1G 2W1 \\ Review of Myers et al.
}

Neuronal migration is an essential process in brain development. Asymmetric cell division takes place within the developing brain, as neural stem cell precursors simultaneously generate new neurons and renew the stem cell population (Gönczy, 2008). After exiting the cell cycle, newly formed neurons migrate to form the developing cerebral cortex (Ayala et al., 2007). Neurons arise from two distinct germinal zones. Excitatory neurons are generated in the subventricular zone (SVZ) and migrate radially to the cortical plate, while inhibitory neurons are generated in the medial and caudal ganglionic eminences and migrate tangentially (Xu el al., 2004). Proper migration and integration of the cortical interneuron population is essential for the formation of functional cortical circuitry within the brain. Migrating interneurons are separated into an upper marginal zone (MZ) stream and a lower subventricular zone/ intermediate zone (SVZ/IZ) stream as they move to the cerebral cortex, where terminal differentiation occurs (Huang, 2009). While the molecular events governing neuronal migration via chemoattraction and chemorepulsion are well characterized (Marín et al., 2001; Flames

Received Aug. 4, 2014; revised Sept. 3, 2014; accepted Sept. 8, 2014. We thank Dr John Vessey for his editorial assistance.

The authors declare no competing financial interests.

Correspondence should be addressed to J. Jacob Riches. Department of

Molecular and Cellular Biology, College of Biological Science, University of Guelph, Guelph, Ontario, Canada, N1G 2W1. E-mail: richesj@uoguelph.ca. DOI:10.1523/JNEUROSCI.3222-14.2014

Copyright $\odot 2014$ the authors $\quad 0270-6474 / 14 / 3414165-02 \$ 15.00 / 0$ et al., 2004), the intracellular signals guiding neuronal migration into the cerebral cortex remain under investigation.

One likely mediator of neuronal migration is the c-Jun N-terminal protein kinase (JNK) pathway. Mammalian JNKs are encoded by the Jnk1, Jnk2, and Jnk3 genes (Kuan et al., 1999). They belong to the mitogen-activated protein kinase (MAPK) family of nonreceptor kinases and they phosphorylate components of multiple signaling pathways (Ayala et al., 2007). JNKs mediate neuronal cell death in response to stress or injury (Sun et al., 2007). They are also crucial for embryonic development: combinatorial deletion of mammalian Jnk1 and Jnk2 leads to dysregulation of apoptosis in the forebrain and produces a nonviable embryo (Kuan et al., 1999). In contrast, loss of Jnk1 alone affects the migration of cortical projection neurons (Hirai et al., 2006).

To investigate the significance of JNK activity in interneuron migration, Myers et al. (2014) made use of pan-JNK chemical inhibitors in live brain slice cultures from transgenic Dlx 5/6 Cre-IRES-EGFP (Dlx5/6-CIE) mice, which express EGFP and Cre in newly born interneurons. E12.5 brain slices were cultured for $24 \mathrm{~h}$ in the presence of JNK inhibitors. Under these conditions, a significantly lower proportion of Dlx $5 / 6+$ interneurons was found in the distal portions of the cortical rudiment compared with controls, and a higher proportion was located at the cortical entry zone (Myers et al., 2014, their Figs. $2 B-H, 3 A, B)$. These results were supported by the use of knock-out mice. While cryosections from Jnk2 $2^{-1-}$ mice stained for an interneuronal marker showed no observable defect in interneuron migration relative to controls at E13.5, Jnk1 $1^{-1-}$ mice had a significantly lower proportion of interneurons in the distal portions of the cortical rudiment and a higher proportion at the cortical entry zone (Myers et al., 2014, Figs. 5C, $G, 6 B, F)$. Furthermore, when Myers et al. (2014) combined $J n k 1^{-/-}$with $J n k 2^{+/-}$mice, there was a modest yet significant exacerbation of the $J n k^{-/-}$phenotype in caudal but not rostral portions of the developing cortex (Myers et al., 2014, their Fig. $5 D, H)$. This indicates that Jnk2, although unnecessary for tangential migration, may play a compensatory role in the absence of Jnk1.

As mice lacking both Jnk1 and Jnk2 are embryonic lethal (Kuan et al., 1999), a more sophisticated approach is required to study the consequences of $J n k 1$ and Jnk2 loss in cortical interneurons. Dlx5/6CIE mice express interneuron-specific Cre, allowing ablation of floxed genes in conditional knock-out mice. $J n k 1^{f l f l}$ mice were used in a Jnk2 $2^{-/-}$background to generate mice that are $J n k 2^{-/-}$and whose interneurons lack both $J n k 1$ and $J n k 2$, yet survive to maturity. The researchers demonstrated that cortical interneurons have a cell-intrinsic requirement for Jnk1 signaling to migrate appropriately (Myers et al., 2014, their Fig. $7 B, F$ ).

To obtain a more refined understanding of the migratory defects of interneurons lacking Jnk1 and Jnk2, the authors 
examined the radial distribution of interneurons in the cortical rudiment, the characteristics of the MV and SVZ/IZ streams, and cellular morphology. The distribution of Dlx5/6-CIE; Ink $1^{f l / f l} ;$ Jnk2 $2^{-/-}$interneurons indicated that the SVZ/IZ stream was more dispersed and loosely organized than in controls. Many interneuronal cell bodies lacked visible leading processes, and the somata of several processes were not visible in the plane of section (Myers et al., 2014, their Fig. 9I), suggesting that some Jnk1- and Jnk2deficient interneurons were traveling against the plane of section and the appropriate SVZ/IZ stream. While the proportion of interneurons in the $\mathrm{MZ}$ stream was unperturbed in the Dlx5/6-CIE;Jnk $1^{f l f l}$; $J n k 2^{-/-}$mice, they formed a patchy stream with several gaps (Myers et al., 2014, their Fig. 9H). In contrast, the interneurons of control mice traveled in a coherent stream at the MZ (Myers et al., 2014, their Fig. 9D). Finally, morphological defects were described in Jnk1- and Jnk2-null interneurons cultured in vitro: processes were more highly branched than those of control interneurons (Myers et al., 2014, their Fig. 9J, K).

Future research will be necessary to determine the degree to which Jnk2 plays a compensatory role in the absence of Jnk1 activity. Myers et al. (2014) scratched the surface of this problem, showing that when the $J n k 1^{-1-}$ genotype was combined with $J n k 2^{+/-}$, there was a modest yet significant exacerbation of the $J n k 1^{-/-}$ phenotype in caudal but not rostral portions of the developing cortex (Myers et al., 2014, their Fig. 5D, $H$ ). These data beg the question of whether variation exists in the expression and/or activity of Jnk1 and/or Jnk2 in interneurons from the rostral versus caudal portions of the cortex. Had Myers et al. (2014) included a second experimental group of $J n k 1^{f l f f} ; J n k 2^{+/-}$ mice in their $J n k 1$ conditional knock-out experiments, they could have addressed in greater detail the question of compensatory Jnk2 activity. This would have been particularly informative in the experiments presented in Figure 9 of their publication. Without an experimental group of $J n k 1^{f l / f l} ; J n k 2^{+/-}$mice, it is not possible to determine whether the severity of the phenotypes described in their Figure 9 as affecting the radial distribution of interneurons in the cortical rudiment, the disorder of the MV and SVZ/IZ streams, and cellular morphology associated with the Dlx5/6-CIE;Jnk1 ${ }^{f l / f l} ; J n k 2^{-/-}$genotype are due to loss of both Jnk1 and Jnk2 or simply due to loss of Jnk1 activity. These experiments would have clarified the significance of the proposed compensatory role of Jnk2 in interneuron migration in the absence of Jnk1.

As noted above, Jnk1 and Jnk2 double knock-out mice are embryonic lethal. These Jnk1 $1^{-1-} ; n k k 2^{-/-}$mice die between E11.5 and E12.5 due to severe dysregulation of apoptosis in the brain (Kuan et al., 1999). This includes increased apoptosis in the forebrain, the portion of the developing brain that will produce the medial and caudal ganglionic eminences and the vertebral cortex. Given the temporal and spatial proximity of the apoptosis seen in Jnk $1^{-/-}$; Ink $2^{-/-}$embryos and the onset of interneuron migration, it would be interesting to know how loss of $J n k 1$ alone and in combination with Jnk2 affects the rate of apoptosis in migrating interneurons. Indeed, in many of the experiments performed by Myers et al. (2014), indistinguishable results might be expected from interneurons with a migratory defect and interneurons prone to apoptosis while traversing the cortical rudiment. In addition, an additive negative effect would likely be seen in interneurons that are both prone to apoptosis and have a migratory defect. To untangle the relative contributions of these deficits in survival and migration, one would need to address the rate of apoptosis directly.

Myers et al. (2014) left the question of Jnk3's role in cortical interneuron migration to future studies. Jnk3 may be of significant interest because, unlike the ubiquitously expressed Jnk1 and Jnk2, Jnk3 is found predominantly in the brain (Sun et al., 2007). The data reported by Myers et al. (2014) on the effect of panJNK inhibitors, which simultaneously inhibit Jnk1, Jnk2, and Jnk3 (Myers et al., 2014, their Fig. 2), suggest a role for Jnk3 in interneuronal migration. Though it is difficult to directly compare these results with those of knock-outs, as the experiments were not performed identically, it is worth noting that the inhibitors produced more significant defects in distribution of interneurons in the cortical rudiment than genetic ablation of both Jnk1 and Jnk2 (Myers et al., 2014, their Fig. 7).

The results of Myers et al. (2014) demonstrate that JNK plays a key role in the migration of interneurons to the cerebral cortex. Use of ex vivo pharmacological JNK inhibition showed that interneurons migrate with decreased velocity and inefficient directionality when JNK activity is lost. In vivo knock-out models reinforced these findings, with deletion of $J n k 1$ leading to slower migration of cortical interneurons and inefficient, poorly directed movement into the cerebral cortex. In regards to Jnk2, this effect on migration was only observed in Jnk1-null conditions, suggesting a potential compensatory role for Jnk 2 in the absence of Jnk1. These results provide evidence that JNK signaling, especially that of Jnk1, is crucial for proper neuronal migration. However, to fully understand the role of JNKs, the rate of apoptosis in migrating cortical interneurons should be examined. Future research regarding the role of Jnk3 in neuronal migration will also help to further elucidate the role in which this signaling pathway mediates the movement of cortical interneurons into the cerebral cortex. Nonetheless, the research of Myers et al. (2014) demonstrates that the JNK pathway plays a vital role in regulating interneuronal migration during development, and thus enhances our understanding of the molecular events surrounding the formation and functional connectivity of the mammalian brain.

\section{References}

Ayala R, Shu T, Tsai LH (2007) Trekking across the brain: the journey of neuronal migration. Cell 128:29-43. CrossRef Medline

Flames N, Long JE, Garratt AN, Fischer TM, Gassmann M, Birchmeier C, Lai C, Rubenstein JL, Marín O (2004) Short- and longrange attraction of cortical GABAergic interneurons by neuregulin-1. Neuron 44: 251-261. CrossRef Medline

Gönczy P (2008) Mechanisms of asymmetric cell division: flies and worms pave the way. Nat Rev Mol Cell Bio 9:355-366. CrossRef Medline

Hirai S, Cui DF, Miyata T, Ogawa M, Kiyonari H, Suda Y, Ohno S (2006) The c-jun N-terminal kinase activator dual leucine zipper kinase regulates axon growth and neuronal migration in the developing cerebral cortex. J Neurosci 26:11992-12002. Medline

Huang Z (2009) Molecular regulation of neuronal migration during neocortical development. $\mathrm{Mol}$ Cell Neurosci 42:11-22. CrossRef Medline

Kuan CY, Yang DD, Samanta Roy DR, Davis RJ, Rakic P, Flavell RA (1999) The Jnk1 and Jnk2 protein kinases are required for regional specific apoptosis during early brain development. Neuron 22:667-676. CrossRef Medline

Marín O, Yaron A, Bagri A, Tessier-Lavigne M, Rubenstein JL (2001) Sorting of striatal and cortical interneurons regulated by semaphoring-neuropilin interactions. Science 293:872-875. CrossRef Medline

Myers AK, Meechan DW, Adney DR, Tucker ES (2014) Cortical interneurons require Jnkl to enter and navigate the developing cerebral cortex. J Neurosci 34:7787-7801. CrossRef Medline

Sun Y, Yang T, Xu Z (2007) The JNK pathway and neuronal migration. J Genet Genomics 34:957-965. CrossRef Medline

Xu Q, Cobos I, De La Cruz E, Rubenstein JL, Anderson SA (2004) Origins of cortical interneuron subtypes. J Neurosci 24:2612-2622. CrossRef Medline 